\title{
INFLUÊNCIA DOS FORÓFITOS Dicksonia sellowiana e Araucaria angustifolia SOBRE A COMUNIDADE DE EPÍFITOS VASCULARES EM FLORESTA COM ARAUCÁRIA
}

\author{
Diego Fedrizzi Petry Becker ${ }^{1 *}$, Andressa Müller ${ }^{1}$, Jairo Lizandro Schmitt ${ }^{2}$ \\ ${ }^{1 *}$ Universidade Feevale, Programa de Pós-Graduação em Qualidade Ambiental, Novo Hamburgo, Rio Grande do Sul, Brasil - \\ biologo.diego@yahoo.com.br; andressam@feevale.br \\ ${ }^{2}$ Universidade Feevale, Departamento de Ciências Biológicas, Novo Hamburgo, Rio Grande do Sul, Brasil - jairols@ feevale.br
}

Recebido para publicação: 10/07/2014 - Aceito para publicação: 06/03/2015

\begin{abstract}
Resumo
Foi verificada a influência da umidade e do $\mathrm{pH}$ do substrato de Araucaria angustifolia (Bertol.) Kuntze e de Dicksonia sellowiana Hook. sobre a riqueza, composição e estrutura comunitária dos epífitos vasculares no interior da Floresta Ombrófila Mista. Para o estudo da comunidade epifítica foram selecionados 20 forófitos de A. angustifolia e de D. sellowiana, inseridos em um hectare de Floresta Ombrófila Mista. Os forófitos foram divididos igualmente em quatro intervalos de um metro de altura cada. A. angustifolia apresentou 20 espécies, enquanto que $D$. sellowiana 11 . A média de espécies por forófito foi significativamente maior em $D$. sellowiana que em A. angustifolia. Os valores de umidade e pH diferiram estatisticamente entre os tipos forofíticos e a maior relação foi observada entre umidade e riqueza de samambaias epifíticas. A análise de coordenadas principais evidenciou agrupamentos bem definidos para os tipos forofíticos e heterogeneidade florística entre os intervalos de altura. Blechnum acutum (Desv.) Mett. ocupou ambos os forófitos e apresentou os maiores valores de importância, demonstrando alta tolerância ecológica. Os resultados indicam que a umidade foi o fator que apresentou maior influência na estrutura da comunidade epifítica, especialmente para as samambaias.
\end{abstract}

Palavras-chave: Epifitismo; Floresta Ombrófila Mista; conífera; samambaia arborescente.

\begin{abstract}
Dicksonia sellowiana and Araucaria angustifolia phorophyte influence on the vascular epiphyte community in Araucaria Forest. We analyzed the influence of moisture and $\mathrm{pH}$ substrate of Araucaria angustifolia (Bertol.) Kuntze e Dicksonia sellowiana Hook. on the richness, composition and community structure of vascular epiphytes within the Araucaria Forest. In order to study the epiphytic community, 20 phorophytes of A. angustifolia and D. sellowiana were selected in one hectare of Araucaria Forest. The phorophytes were divided equally into four intervals of one meter each. Araucaria angustifolia showed 20 species, while D. sellowiana 11. Means of species per host tree were significantly higher in D. sellowiana than for A. angustifolia. The moisture and $\mathrm{pH}$ of phorophyte types differ statistically and the most relation was observed between moisture and epiphytic ferns richness. Principal coordinates analysis showed well-defined groupings for phorophyte types and floristic heterogeneity among height intervals. Blechnum acutum (Desv.) Mett. occupied both phorophytes and had the highest importance values, demonstrating high ecological tolerance. The results show that moisture was the factor with the highest influence in the epiphytic community structure, especially for the ferns.
\end{abstract}

Keywords: Epiphytism; Mixed Ombrophilous Forest; conifer; tree ferns.

\section{INTRODUÇÃO}

A flora epifítica é responsável por parte significativa da diversidade das florestas, representando cerca de 10\% de todas as plantas vasculares (KRESS, 1986). A riqueza, composição e abundância de epífitos dependem de características do substrato disponível para o seu estabelecimento, entre outros fatores (WAGNER et al., 2015). Os forófitos apresentam diferenças físicas e químicas que podem variar 
no que se refere à arquitetura, diâmetro, rugosidade da superfície, potencial hidrogeniônico $(\mathrm{pH})$ e umidade (JOHANSSON, 1974, MEHLTRETER et al., 2005).

Segundo o Instituto Brasileiro de Geografia e Estatística (IBGE) (2012) a Floresta Ombrófila Mista, também conhecida como Floresta com Araucária, é uma unidade fitoecológica do bioma Floresta Atlântica e distribui-se no Planalto Sul-Brasileiro, em altitudes médias de 600 a 800 m, podendo atingir mais de $1000 \mathrm{~m}$ em algumas áreas. Além disso, possui como elemento característico a presença de Araucaria angustifolia (Bertol.) Kuntze. e no sub-bosque Dicksonia sellowiana Hook., às quais lhe conferem um aspecto fisionômico bastante particular. No Brasil, essa formação florestal encontra-se reduzida a aproximadamente $12 \%$ da área original (FUNDAÇÃO SOS MATA ATLÂNTICA; INSTITUTO NACIONAL DE PESQUISAS ESPACIAIS (INPE), 2008) e abriga uma importante diversidade de plantas vasculares, incluindo espécies epifíticas (KERSTEN; KUNIYOSHI, 2009).

Araucaria angustifolia é uma conífera com distribuição geográfica restrita à América Latina (MISSOURI BOTANICAL GARDEN, 2014), com fuste retilíneo, que pode atingir de 20 a $50 \mathrm{~m}$ de altura e diâmetro de até dois metros (LORENZI, 2008). As aberturas na casca de A. angustifolia podem favorecer a germinação de espécies de epífitos devido ao aumento na disponibilidade de água nessas regiões (REINERT; FONTOURA, 2008). A intensa exploração extrativista da madeira no passado, bem como da sua semente até os dias de hoje, levou a espécie a ser incluída no Livro Vermelho da Flora do Brasil (MARTINELLI; MORAES, 2013).

Dicksonia sellowiana constitui um microhabitat distinto para a existência de espécies epifíticas, incluindo algumas exclusivas ou que crescem preferencialmente sobre elas (SCHMITT et al., 2005). A espécie, conhecida popularmente como Xaxim, é uma samambaia arborescente com distribuição também restrita à América Latina (MISSOURI BOTANICAL GARDEN, 2014). Seu fuste pode atingir até $10 \mathrm{~m}$ de altura, $1 \mathrm{~m}$ de diâmetro, não apresenta crescimento secundário e é composto por densa camada de raízes adventícias que pode ser utilizada como um eficiente suporte mecânico por algumas espécies de epífitos (TRYON; TRYON, 1982). A capacidade de retenção de umidade, o acúmulo de matéria orgânica e a consequente eficiência desse substrato para o cultivo de plantas ornamentais, levaram a espécie a grande exploração ilegal no passado, resultando também na sua inclusão no Livro Vermelho da Flora do Brasil (MARTINELLI; MORAES, 2013).

Poucos estudos analisaram a variação da riqueza de epífitos entre samambaias arborescentes e espécies arbóreas. Mehltreter et al. (2005), no México, analisaram a influência do substrato em diferentes forófitos em Floresta Tropical Úmida Montana, obtendo as maiores médias de espécies em samambaias arborescentes, quando comparado às angiospermas arbóreas. Nesse estudo, apenas a umidade do forófito se relacionou com a abundância de epífitos. Moran et al. (2003), na Costa Rica, registraram maior riqueza e média significativamente maior de espécies nos forófitos de samambaias arborescentes em relação à angiospermas arbóreas, no entanto sem investigar a influência das características dos substratos.

A semelhança na verticalidade do fuste de A. angustifolia e de $D$. sellowiana permite a análise da influência do substrato na estrutura da comunidade epifítica desses forófitos. Considerando isso, o objetivo do estudo foi verificar a influência do teor de umidade e do $\mathrm{pH}$ do substrato de A. angustifolia e de $D$. sellowiana sobre a riqueza, a composição e a estrutura comunitária dos epífitos vasculares no interior da Floresta Ombrófila Mista.

\section{MATERIAL E MÉTODOS}

\section{Área de estudo}

O trabalho foi conduzido em remanescente de Floresta Ombrófila Mista, na Floresta Nacional de São Francisco de Paula (29²5'14.06”'S; 50²3'39.18”O; 900 m de altitude), município de São Francisco de Paula, Rio Grande do Sul, Brasil. Segundo a classificação de Köppen, o clima da região é do tipo Cfb, isto é, mesotérmico, superúmido, com verão brando e inverno frio. De acordo com o Instituto Chico Mendes de Conservação da Biodiversidade (ICMBIO) (2012), temperatura média anual é de $14,5{ }^{\circ} \mathrm{C}$ e a pluviosidade superior a $2.000 \mathrm{~mm}$ por ano.

\section{Forófitos}

Para o estudo da comunidade epifítica foram selecionados, por meio de sorteio, 20 forófitos de Araucaria angustifolia e de Dicksonia sellowiana, inseridos em um hectare de Floresta Ombrófila Mista e 
com fuste de, no mínimo, quatro metros. Para a padronização da amostragem os primeiros quatro metros de fuste foram divididos em quatro intervalos verticais de um metro cada.

\section{Florística}

As espécies foram analisadas quanto à ocorrência nos tipos forofíticos e nos intervalos de altura. O registro das plantas se deu pela observação direta nos forófitos e coleta de espécime representativo fértil. Esse material testemunho foi herborizado, segundo técnicas usuais de laboratório, e incluído na coleção do Herbarium Anchieta (PACA). A identificação dos epífitos se deu com o auxílio de bibliografia especializada, comparação com material de herbário e consulta a especialistas. Foram utilizadas categorias ecológicas para verificar a presença de espécies características, acidentais e secundárias, conforme Benzing (1990): holoepífitos habituais (HAB): não estabelecem relação com o solo e completam todo o seu ciclo de vida no ambiente epifítico; holoepífitos facultativos (FAC): podem ser tanto epífitos, rupícolas ou terrícolas; holoepífitos acidentais (ACI): são encontrados ocasionalmente no ambiente epifítico; e hemiepífitos secundários (HMS): após germinar no solo, atingem o forófito e perdem a ligação com o solo.

\section{Potencial hidrogeniônico e umidade do substrato}

$\mathrm{O}$ pH foi determinado a partir da adaptação do método adotado por Johansson (1974), no qual foi retirada uma amostra de três a cinco centímetros quadrados de superfície, com até dois milímetros de espessura de cada intervalo de altura. O material coletado foi colocado em um frasco esterilizado, com $50 \mathrm{ml}$ de água destilada, misturado por 30 segundos, permanecendo mais dois minutos para estabilização e em seguida realizada a medição do $\mathrm{pH}$ final. $\mathrm{O}$ equipamento utilizado foi um potenciômetro digital portátil (PHTEK®) devidamente calibrado.

Da mesma forma, a umidade do substrato foi obtida a partir da retirada de três a cinco centímetros quadrados de superfície e com até dois milímetros de espessura de cada intervalo de altura. As amostras foram acondicionadas separadamente em embalagens plásticas e levadas ao laboratório para obtenção da massa inicial em uma balança analítica Mettler Toledo® modelo AL204 e posterior secagem em estufa a $70^{\circ} \mathrm{C}$. Para obtenção dos teores de umidade, o resultado entre a diferença da massa inicial e final, foi dividido pela massa inicial.

\section{Parâmetros fitossociológicos}

Para caracterização da estrutura comunitária dos epífitos nas duas espécies forofíticas foi calculada a frequência relativa das espécies nos forófitos e nos intervalos de altura. Para cada espécie, foi atribuída uma nota de cobertura (1, 3, 5, 7 ou 10) no respectivo intervalo, segundo Kersten e Waechter (2011). A partir do somatório das notas de cobertura, foi calculada a cobertura relativa por intervalo de cada espécie. O valor de importância específico (VIe) resultou da média aritmética da soma das frequências relativas nos forófitos, nos intervalos e da cobertura relativa.

\section{Análises estatísticas}

Médias de riqueza total, de samambaias e angiospermas epifíticas, bem como o teor de umidade e o $\mathrm{pH}$ dos dois tipos forofíticos, foram submetidos ao teste de normalidade Shapiro-Wilk. Os dados que atenderam ao pressuposto de normalidade foram comparados por meio do teste $\mathrm{t}$ de Student a 5\% de probabilidade e, aqueles que não apresentaram distribuição normal foram analisados pelo teste não paramétrico de Mann-Whitney a 5\% de probabilidade. A relação entre a riqueza de espécies nos intervalos, com pH e umidade do substrato, foi verificada pelo teste não paramétrico de correlação de postos de Spearman $\left(r_{s}\right)$, em nível de significância de 5\%. Foram adotados os valores de referência que qualificam as correlações de acordo com Callegari-Jacques (2007). Essas análises foram realizadas por meio do programa estatístico SPSS versão 20.0.

Os dados de presença ou ausência das espécies nos intervalos de altura dos forófitos foram utilizados na Análise de Coordenadas Principais (PCoA) com o índice de Dice-Sorensen para verificar a similaridade dos forófitos e dos intervalos de altura. A Análise de Componentes Principais (PCA) foi utilizada na representação das relações entre o $\mathrm{pH}$, umidade, riqueza de espécies, samambaias e angiospermas por forófitos, com a matriz de correlação. Ambos testes foram realizados por meio do programa Paleontological Statistics Software Package for Education and Data Analysis (PAST), versão 3.01. 


\section{RESULTADOS}

\section{Florística}

No levantamento florístico foram registradas 11 espécies em Dicksonia sellowiana e 20 em Araucaria angustifolia (Tabela 1). Dessas, oito e 15 foram samambaias epifíticas, nos respectivos forófitos. Nove forófitos de D. sellowiana e 14 de A. angustifolia não apresentaram angiospermas epifíticas. Apenas quatro espécies foram comuns aos dois tipos forofíticos. No total, foram registradas 27 espécies, distribuídas em 18 gêneros e nove famílias. Samambaias contribuíram com 20 espécies e angiospermas com sete.

Tabela 1. Espécies de epífitos vasculares sobre Dicksonia sellowiana (Ds) e Araucaria angustifolia (Aa), com as respectivas categorias ecológicas, na Floresta Nacional de São Francisco de Paula, RS, Brasil. Holoepífito habitual (HAB); Holoepífito facultativo (FAC); Holoepífito acidental (ACI); Hemiepífito secundário (HMS).

Table 1. Species of vascular epiphytes on Dicksonia sellowiana (Ds) and Araucaria angustifolia (Aa), with its ecological categories, in the National Forest of São Francisco de Paula, RS, Brazil. Habitual holoepiphyte (HAB); Facultative holoepiphyte (FAC); Accidental holoepiphyte (ACI); secondary hemiepiphyte (HMS).

\begin{tabular}{|c|c|c|c|}
\hline Família/Espécie & Ds & Aa & Categoria Ecológica \\
\hline \multicolumn{4}{|l|}{ ASPLENIACEAE } \\
\hline Asplenium gastonis Fée & & $\mathrm{X}$ & FAC \\
\hline Asplenium harpeodes Kunze & $\mathrm{X}$ & & HAB \\
\hline Asplenium serra Langsd. \& Fisch. & & $\mathrm{X}$ & FAC \\
\hline \multicolumn{4}{|l|}{ BLECHNACEAE } \\
\hline Blechnum acutum (Desv.) Mett. & $\mathrm{X}$ & $\mathrm{X}$ & HMS \\
\hline \multicolumn{4}{|l|}{ BROMELIACEAE } \\
\hline Tillandsia aeranthos (Loisel.) L.B. Sm. & & $\mathrm{X}$ & HAB \\
\hline Tillandsia recurvata $(\mathrm{L}.) \mathrm{L}$. & & $\mathrm{X}$ & HAB \\
\hline Vriesea gigantea Gaudich. & & $\mathrm{X}$ & HAB \\
\hline \multicolumn{4}{|l|}{ CYPERACEAE } \\
\hline Cyperaceae sp1 & $\mathrm{X}$ & & ACI \\
\hline Cyperaceae sp2 & $\mathrm{X}$ & $\mathrm{X}$ & $\mathrm{ACI}$ \\
\hline \multicolumn{4}{|l|}{ DRYOPTERIDACEAE } \\
\hline Elaphoglossum sellowianum (Klotzsch ex Kuhn) T. Moore & & $\mathrm{X}$ & FAC \\
\hline Lastreopsis amplissima (C. Presl.) Tindale & & $\mathrm{X}$ & $\mathrm{ACI}$ \\
\hline Polystichum montevidense (Spreng.) Rosenst. & & $\mathrm{X}$ & ACI \\
\hline Rumohra adiantiformis (G. Forst.) Ching & & $\mathrm{X}$ & ACI \\
\hline \multicolumn{4}{|l|}{ HYMENOPHYLLACEAE } \\
\hline Hymenophyllum pulchellum Schltdl. \& Cham. & $\mathrm{X}$ & & $\mathrm{HAB}$ \\
\hline Polyphlebium angustatum (Carmich.) Ebihara \& Dubuisson & $\mathrm{X}$ & & HAB \\
\hline Trichomanes anadromum Rosenst. & $\mathrm{X}$ & & HAB \\
\hline \multicolumn{4}{|l|}{ PIPERACEAE } \\
\hline Peperomia catharinae Miq. & $\mathrm{X}$ & & $\mathrm{HAB}$ \\
\hline Peperomia trineura Miq. & & $\mathrm{X}$ & HAB \\
\hline \multicolumn{4}{|l|}{ POLYPODIACEAE } \\
\hline Campyloneurum austrobrasilianum (Alston) de la Sota & $\mathrm{X}$ & $\mathrm{X}$ & HAB \\
\hline Campyloneurum nitidum (Kaulf.) C. Presl. & & $\mathrm{X}$ & HAB \\
\hline Microgramma squamulosa (Kaulf.) de la Sota & & $\mathrm{X}$ & HAB \\
\hline Pecluma pectinatiformis (Lindm.) M.G. Price & $\mathrm{X}$ & $\mathrm{X}$ & НАВ \\
\hline Pleopeltis astrolepis (Liebm.) E. Fourn. & & $\mathrm{X}$ & HAB \\
\hline Pleopeltis hirsutissima (Raddi) de la Sota & & $\mathrm{X}$ & HAB \\
\hline Pleopeltis pleopeltidis (Fée) de la Sota & & $\mathrm{X}$ & HAB \\
\hline Pleopeltis pleopeltifolia (Raddi) Alston & & $\mathrm{X}$ & HAB \\
\hline \multicolumn{4}{|l|}{ PTERIDACEAE } \\
\hline Vittaria lineata $(\mathrm{L}.) \mathrm{Sm}$. & $\mathrm{X}$ & & HAB \\
\hline
\end{tabular}

FLORESTA, Curitiba, PR, v. 45, n. 4, p. 781 - 790, out. / dez. 2015. 
Hymenophyllaceae foi a família com maior riqueza em D. sellowiana, com três espécies, correspondendo a $27 \%$ do total nesse grupo de forófitos. Polypodiaceae apresentou maior riqueza nos forófitos de A. angustifolia com oito espécies (40\% do total). D. sellowiana apresentou oito holoepífitos habituais (73\%), dois acidentais e um hemiepífito secundário, enquanto que em A. angustifolia foram 12 holoepífitos habituais (60\%), quatro acidentais, três facultativos e um hemiepífito secundário. A PCoA explicou 85,96\% da variação e evidenciou a heterogeneidade da composição florística dos forófitos, bem como dos intervalos de altura (Figura 1).

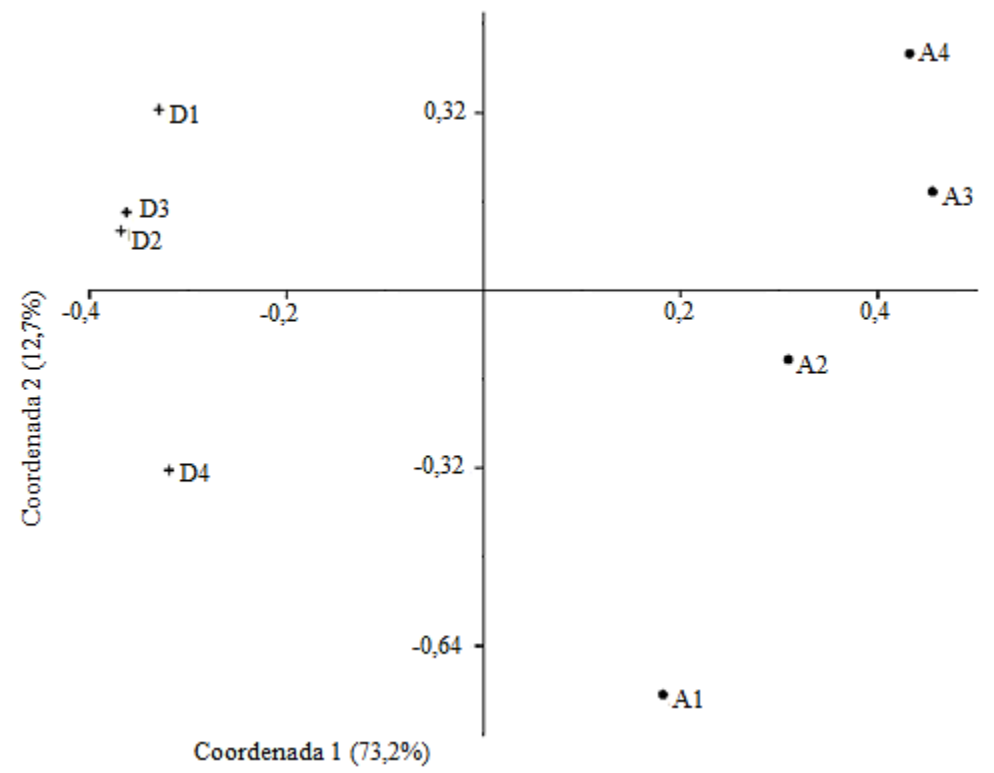

Figura 1. Análise de Coordenadas Principais (PCoA) da composição de epífitos vasculares nos intervalos de altura $(1,2,3$ e 4$)$ dos forófitos de Dicksonia sellowiana $(+D)$ e Araucaria angustifolia $(\bullet A)$ da Floresta Nacional de São Francisco de Paula, RS, Brasil.

Figure 1. Principal Coordinates Analysis (PCoA) of the composition of vascular epiphytes in height intervals (1, 2, 3 and 4) of Dicksonia sellowiana (+D) and Araucaria angustifolia $(\cdot A)$ phorophytes of the National Forest of São Francisco de Paula, RS, Brazil.

Embora Araucaria angustifolia tenha apresentado maior riqueza total, o mesmo não foi observado para a média de espécies por forófito (Tabela 2). As médias de riqueza total e de samambaias epifíticas diferiram estatisticamente entre os tipos forofíticos, enquanto que a média de angiospermas não diferiu.

Tabela 2. Médias de riqueza total, de samambaias e de angiospermas epifíticas em Dicksonia sellowiana (Ds) e Araucaria angustifolia (Aa) na Floresta Nacional de São Francisco de Paula, RS, Brasil.

Table 2. Averages of total, epiphytic ferns and angiosperms richness in Dicksonia sellowiana (Ds) and Araucaria angustifolia (Aa) in the National Forest of São Francisco de Paula, RS, Brazil.

\begin{tabular}{lccc}
\hline Forófito & $\begin{array}{c}\text { Total } \\
\text { (Espécies forófitos }^{\mathbf{1}} \text { ) }\end{array}$ & $\begin{array}{c}\text { Samambaias } \\
\text { (Espécies forófitos }^{-\mathbf{1}} \text { ) }\end{array}$ & $\begin{array}{c}\text { Angiospermas } \\
\text { (Espécies forófitos }^{-1} \text { ) }\end{array}$ \\
\hline Ds $(\mathrm{n}=20)$ & $3,6 \pm 1,5$ & $3,0 \pm 1,3$ & $0,6 \pm 0,6$ \\
Aa $(\mathrm{n}=20)$ & $2,2 \pm 1,2$ & $1,8 \pm 1,0$ & $0,4 \pm 0,6$ \\
& $\mathrm{U}=75,5$ & $\mathrm{U}=83,5$ & $\mathrm{U}=152,5$ \\
& $\mathrm{P}<0,001$ & $\mathrm{P}=0,002$ & $\mathrm{P}=0,198$ \\
\hline
\end{tabular}

\section{Potencial hidrogeniônico e umidade do substrato}

Dicksonia sellowiana apresentou $\mathrm{pH}$ significativamente mais ácido $(\mathrm{t}=-7,74 ; \mathrm{P}<0,001)$ que

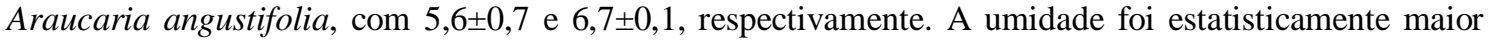
$(\mathrm{U}<0,01 ; \mathrm{P}<0,001)$ em D. sellowiana $(74,4 \pm 6,7 \%)$ do que em A. angustifolia $(30,2 \pm 5,8 \%)$. Riqueza total 
por intervalo apresentou relação negativa moderada com o $\mathrm{pH}(\mathrm{r}=-0,38 ; \mathrm{P}=0,015)$ e positiva moderada com a umidade do substrato $(\mathrm{r}=0,58 ; \mathrm{P}=0,009)$. Riqueza de samambaias apresentou relação negativa moderada com o $\mathrm{pH}(\mathrm{r}=-0,45 ; \mathrm{P}=0,003)$ e positiva forte com umidade $(\mathrm{r}=0,62 ; \mathrm{P}=0,005)$. Riqueza de angiospermas não se relacionou com $\mathrm{pH}(\mathrm{r}=0,05 ; \mathrm{P}=0,739)$ e umidade do substrato $(\mathrm{r}=0,10 ; \mathrm{P}=0,674)$.

Os dois primeiros eixos da PCA explicaram $74,7 \%$ da variação e evidenciaram uma separação dos grupos forofíticos em função das variáveis analisadas (Figura 2). Essa separação ocorreu principalmente no componente $1(49,3 \%)$, onde a riqueza de espécies foi a variável mais relacionada $(0,57)$, seguida da riqueza de samambaias $(0,51)$ e do $\mathrm{pH}(-0,42)$. No componente $2(25,4 \%)$ a variável com maior relação foi a umidade do substrato $(-0,64)$, seguida do $\mathrm{pH}(0,52)$. Em geral, os forófitos de $D$. sellowiana estiveram associados aos maiores teores de umidade, ao menor $\mathrm{pH}$ e à maior riqueza por indivíduo, enquanto que A. angustifolia foi mais relacionada ao maior valor de $\mathrm{pH}$, à menor umidade e ao menor número de espécies por unidade amostral.

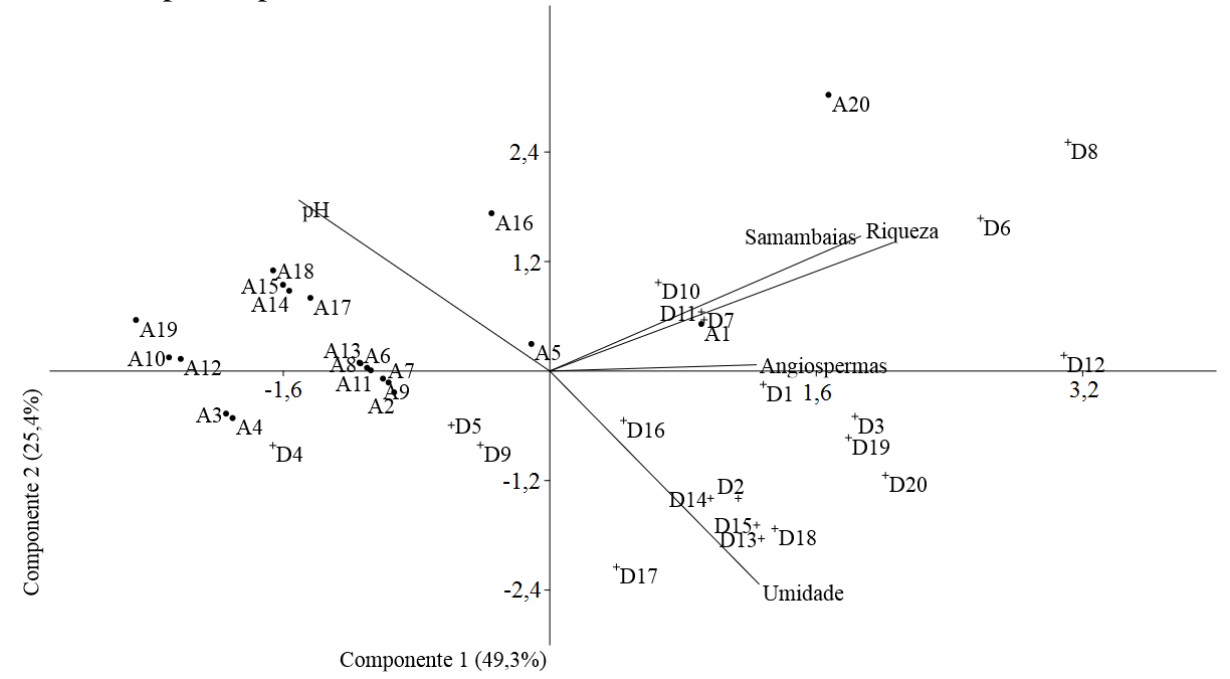

Figura 2. Análise de Componentes Principais (PCA) dos forófitos de Dicksonia sellowiana (+) e Araucaria angustifolia $(\bullet)$ e suas relações com $\mathrm{pH}$, umidade, riqueza total, de samambaias e de angiospermas, na Floresta Nacional de São Francisco de Paula, RS, Brasil.

Figure 2. Principal Components Analysis (PCA) of Dicksonia sellowiana (+) and Araucaria angustifolia $(\bullet)$ phorophytes and their relationship with $\mathrm{pH}$, moisture, total richness, ferns and angiosperms in the National Forest of São Francisco de Paula, RS, Brazil.

\section{Parâmetros fitossociológicos}

Em Dicksonia sellowiana, o maior VIe foi observado para Blechnum acutum, com ocorrência em $100 \%$ dos forófitos, seguido por Trichomanes anadromum, registrado em $90 \%$ desses. Quatro espécies ocorreram em apenas um forófito e um intervalo de altura, obtendo assim os menores VIe (Tabela 3).

Em Araucaria angustifolia, o maior VIe também foi observado para Blechnum acutum, seguido de Pleopeltis hirsutissima, ambos com ocorrência em 30\% dos forófitos. O total de 10 espécies ocorreu em apenas um forófito e um intervalo de altura, obtendo assim os menores VIe (Tabela 4).

\section{DISCUSSÃO}

A menor média de espécies em Araucaria angustifolia pode ser atribuída ao grande número de taxa com baixa frequência nos forófitos e intervalos e à menor cobertura das espécies epifíticas. Além disso, ficou evidente que as samambaias contribuíram significativamente para a maior média de riqueza em Dicksonia sellowiana. A umidade é fundamental para o sucesso reprodutivo das samambaias epifíticas, o que indica que a maior umidade em $D$. sellowiana foi o fator que mais contribuiu para as diferenças nas médias registradas. Similarmente, Johansson (1974), na África, registrou espécies epifíticas, principalmente samambaias, em Cyathea manniana Hook., que também apresenta manto de raízes adventícias. $\mathrm{O}$ autor não observou flora epifítica em $C$. camerooniana Hook., espécie sem o manto de raízes, o que pode estar relacionado à menor capacidade de acúmulo de água nesses forófitos. 
Tabela 3. Estrutura comunitária de epífitos vasculares sobre Dicksonia sellowiana na Floresta Nacional de São Francisco de Paula, RS, Brasil, em ordem decrescente do valor de importância específico (VIe). Número de forófitos com a espécie (nf); Número de intervalos com a espécie (ni); Frequência relativa da espécie sobre os forófitos (FRf); Frequência relativa da espécie nos intervalos (FRi); Soma das notas de cobertura (NCt); Nota de cobertura relativa da espécie (NCr).

Table 3. Community structure of vascular epiphytes on Dicksonia sellowiana in the National Forest of São Francisco de Paula, RS, Brazil, in descending order of the importance specific value (VIe). Number of phorophytes with the species (nf); Number of intervals with the species (ni); Relative frequency of the species on phorophytes (FRf); Relative frequency of the species in the intervals (FRi); Sum of cover notes (NCt); Relative covering note of specie $(\mathrm{NCr})$.

\begin{tabular}{lccccccc}
\hline Espécies & nf & ni & FRf\% & FRi\% & NCt & NCr\% & VIe \\
\hline Blechnum acutum & 20 & 49 & 28,2 & 35,0 & 206 & 52,6 & 38,6 \\
Trichomanes anadromum & 18 & 51 & 25,4 & 36,4 & 119 & 30,3 & 30,7 \\
Peperomia catharinae & 8 & 12 & 11,3 & 8,6 & 16 & 4,1 & 8,0 \\
Vittaria lineata & 5 & 6 & 7,0 & 4,3 & 16 & 4,1 & 5,1 \\
Polyphlebium angustatum & 6 & 7 & 8,5 & 5,0 & 7 & 1,8 & 5,1 \\
Asplenium harpeodes & 6 & 6 & 8,5 & 4,3 & 7 & 1,8 & 4,8 \\
Pecluma pectinatiformis & 4 & 5 & 5,6 & 3,6 & 11 & 2,8 & 4,0 \\
Campyloneurum austrobrasilianum & 1 & 1 & 1,4 & 0,7 & 3 & 0,8 & 1,0 \\
Cyperaceae sp1 & 1 & 1 & 1,4 & 0,7 & 3 & 0,8 & 1,0 \\
Cyperaceae sp2 & 1 & 1 & 1,4 & 0,7 & 3 & 0,8 & 1,0 \\
Hymenophyllum pulchellum & 1 & 1 & 1,4 & 0,7 & 1 & 0,3 & 0,8 \\
\hline
\end{tabular}

Tabela 4. Estrutura comunitária de epífitos vasculares sobre Araucaria angustifolia na Floresta Nacional de São Francisco de Paula, RS, Brasil, em ordem decrescente do valor de importância específico (VIe). Número de forófitos com a espécie (nf); Número de intervalos com a espécie (ni); Frequência relativa da espécie sobre os forófitos (FRf); Frequência relativa da espécie nos intervalos (FRi); Soma das notas de cobertura (NCt); Nota de cobertura relativa da espécie ( $\mathrm{NCr}$ ).

Table 4. Community structure of vascular epiphytes on Araucaria angustifolia in the National Forest of São Francisco de Paula, RS, Brazil, in descending order of the importance specific value (VIe). Number of phorophytes with the species (nf); Number of intervals with the species (ni); Relative frequency of the species on phorophytes (FRf); Relative frequency of the species in the intervals (FRi); Sum of cover notes ( $\mathrm{NCt}$ ); Relative covering note of specie $(\mathrm{NCr})$.

\begin{tabular}{lccccccc}
\hline Espécies & nf & Ni & FRf\% & FRi\% & NCt & NCr\% & VIe \\
\hline Blechnum acutum & 6 & 8 & 13,6 & 12,9 & 34 & 21,5 & 16,0 \\
Pleopeltis hirsutissima & 6 & 10 & 13,6 & 16,1 & 20 & 12,7 & 14,1 \\
Microgramma squamulosa & 5 & 11 & 11,4 & 17,7 & 19 & 12,0 & 13,7 \\
Tillandsia aeranthos & 3 & 7 & 6,8 & 11,3 & 15 & 9,5 & 9,2 \\
Rumohra adiantiformis & 3 & 3 & 6,8 & 4,8 & 11 & 6,9 & 6,2 \\
Pleopeltis pleopeltidis & 3 & 4 & 6,8 & 6,5 & 8 & 5,1 & 6,1 \\
Campyloneurum nitidum & 2 & 2 & 4,6 & 3,2 & 6 & 3,8 & 3,9 \\
Pecluma pectinatiformis & 2 & 2 & 4,6 & 3,2 & 6 & 3,8 & 3,9 \\
Polystichum montevidense & 2 & 2 & 4,6 & 3,2 & 6 & 3,8 & 3,9 \\
Peperomia trineura & 2 & 3 & 4,6 & 4,8 & 3 & 1,9 & 3,8 \\
Elaphoglossum sellowianum & 1 & 1 & 2,3 & 1,6 & 7 & 4,4 & 2,8 \\
Lastreopsis amplíssima & 1 & 1 & 2,3 & 1,6 & 5 & 3,2 & 2,4 \\
Vriesea gigantea & 1 & 1 & 2,3 & 1,6 & 5 & 3,2 & 2,4 \\
Asplenium serra & 1 & 1 & 2,3 & 1,6 & 3 & 1,9 & 1,9 \\
Cyperaceae sp2 & 1 & 1 & 2,3 & 1,6 & 3 & 1,9 & 1,9 \\
Tillandsia recurvata & 1 & 1 & 2,3 & 1,6 & 3 & 1,9 & 1,9 \\
Asplenium gastonis & 1 & 1 & 2,3 & 1,6 & 1 & 0,6 & 1,5 \\
Campyloneurum austrobrasilianum & 1 & 1 & 2,3 & 1,6 & 1 & 0,6 & 1,5 \\
Pleopeltis astrolepis & 1 & 1 & 2,3 & 1,6 & 1 & 0,6 & 1,5 \\
Pleopeltis pleopeltifolia & 1 & 1 & 2,3 & 1,6 & 1 & 0,6 & 1,5 \\
\hline
\end{tabular}

FLORESTA, Curitiba, PR, v. 45, n. 4, p. 781 - 790, out. / dez. 2015.

Becker, D. F. P. et al.

ISSN eletrônico 1982-4688 / ISSN impresso 0015-3826

DOI: 10.5380/rf.v45i4.37082 
A diferença na riqueza vai de encontro ao registrado por Mehltreter et al. (2005), no México, onde também foi registrado maior média em samambaias arborescentes $(4,3)$ do que em troncos de árvores $(2,9)$. Os autores observaram relação forte e positiva na abundância de indivíduos com o aumento da umidade dos substratos. A umidade do substrato tem sido um dos fatores com maior destaque em levantamentos epifíticos (WAGNER et al., 2015), e pode explicar a abundância e sobrevivência de espécies sensíveis ao ressecamento. O pH registrado por Mehltreter et al. (2005) também diferiu significativamente entre os tipos forofíticos analisados, no entanto não apresentou relação significativa com riqueza e abundância de espécies. Moran et al. (2003), em estudo realizado na Costa Rica, também registraram maior média de samambaias epifíticas sobre cáudices de samambaias arborescentes em relação aos fustes de angiospermas arbóreas.

A heterogeneidade florística do presente estudo deve-se ao baixo número de espécies compartilhadas e às diferenças observadas nos substratos, principalmente no que se refere à umidade. $\mathrm{A}$ maior diferença em relação aos intervalos 1 e 4 pode estar relacionada à fatores extrínsecos dos forófitos (JOHANSSON, 1974), tal como o aumento na radiação solar e diminuição da umidade do ar no sentido base-ápice.

Hymenophyllaceae, que foi a família com maior riqueza em Dicksonia sellowiana, apresenta espécies com adaptações para a sobrevivência em ambientes com baixa luminosidade e altos teores de umidade (PROCTOR, 2012), o que pode ter favorecido a ocupação desse grupo de forófitos. O pH registrado para $D$. sellowiana foi próximo ao valor ótimo obtido para Trichomanes speciosum Willd. (SMYTH et al., 2008), sendo esse mais um fator que pode estar relacionado ao sucesso da família nesses forófitos. Por outro lado, o sucesso de Polypodiaceae em Araucaria angustifolia pode estar relacionado a uma série de adaptações morfoanatômicas e fisiológicas para lidar com baixa disponibilidade de água do substrato, tais como suculência caulinar, poiquiloidria (BENZING, 1990) e, alto índice de esclerofilia e densidade estomática (ROCHA et al., 2013).

Bromeliaceae ocorreu exclusivamente sobre Araucaria angustifolia. Estudos in vitro com essa família utilizam a faixa de $\mathrm{pH}$ registrada como base para seus experimentos (DROSTE et al., 2005). Anacleto et al. (2008) obtiveram maior germinação em pH na faixa de 6,2 a 6,7 para Aechmea nudicaulis (L.) Griseb., sendo esse valor próximo ao encontrado nos forófitos analisados. Avaliando a sobrevivência de Tillandsia geminiflora Brongn. em diferentes substratos, Stringheta et al. (2005) registraram menor taxa de sobrevivência em Dicksonia sellowiana $(\mathrm{pH}=5,75)$. Características morfológicas, como as folhas densamente imbricadas na base, permitem que as bromélias tenham a capacidade de armazenar água (BENZING, 1990) e supostamente ocupar um substrato significativamente menos úmido.

A relação do maior número de espécies em $A$. angustifolia do que em $D$. sellowiana também foi observada por Buzatto et al. (2008), que contabilizaram 17 e nove espécies, na metade inferior dos respectivos forófitos, embora não tenham indicado o número de unidades amostrais. A predominância de holoepífitos habituais também foi observada em outros estudos com D. sellowiana (SCHMITT et al., 2005, BUZATTO et al., 2008), A. angustifolia (BUZATTO et al., 2008) e na mesma formação florestal (KERSTEN; KUNIYOSHI, 2009). Foram registradas espécies com apenas uma ocorrência somente nas regiões mais baixas dos forófitos e classificadas como holoepífitos acidentais. Esse resultado está relacionado ao fato de que os intervalos de altura mais baixos podem apresentar maior acúmulo de matéria orgânica, permitindo a germinação de plantas caracteristicamente terrícolas.

As duas espécies com maior VIe, para ambos os forófitos, são samambaias epifíticas. Blechnum acutum demonstrou possuir alta tolerância ecológica, ocupando ambos os tipos forofíticos e apresentando os maiores VIe. A segunda espécie com maior VIe em Dicksonia sellowiana ocorreu exclusivamente nesses forófitos. A preferência de Trichomanes anadromum por cáudices de samambaias arborescentes já foi relatada em outros estudos (SCHMITT; WINDISCH, 2005), o que indica que o grupo possui uma seletividade em relação ao tipo de substrato.

\section{CONCLUSÕES}

- Umidade do substrato foi o fator que apresentou maior influência na ocupação dos forófitos, em relação ao pH.

- A maior média, frequência e cobertura de espécies em Dicksonia sellowiana confirma que esse forófito apresenta condições ótimas para algumas espécies, como foi o caso de Trichomanes anadromum. 
- O alto número de espécies com ocorrência em apenas um tipo forofítico, evidencia que a retirada de forófitos característicos da Floresta Ombrófila Mista e ameaçados de extinção afeta a comunidade epifítica no que diz respeito à disponibilidade de microhabitat específico, especialmente para os holoepífitos habituais, e mais intensamente para espécies que ocorrem exclusivamente sobre um substrato específico.

\section{AGRADECIMENTOS}

Os autores agradecem à Universidade Feevale pela infraestrutura, à Coordenação de Aperfeiçoamento de Pessoal de Nível Superior (CAPES) pela concessão de bolsa aos dois primeiros autores e à Floresta Nacional de São Francisco de Paula pela autorização para a realização do estudo na Unidade de Conservação.

\section{REFERÊNCIAS}

ANACLETO, A.; NEGRELlE, R. R. B.; KOEHLER, H. S. Germinação de Aechmea nudicaulis (L.) Griseb. (Bromeliaceae) em diferentes substratos alternativos ao pó de xaxim. Acta Scientiarum. Agronomy, v. 30, n. 1, p. 73 - 79, 2008.

BENZING, D. H. Vascular epiphytes. Cambridge: Cambridge University Press. 1990, 354 p.

BUZATTO, C. R.; SEVERO, B. M. A.; WAECHTER, J. L. Composição florística e distribuição ecológica de epífitos vasculares na Floresta Nacional de Passo Fundo, Rio Grande do Sul. Iheringia, Série Botânica, v. 63, n. 2, p. 231 - 239, 2008.

CALLEGARI-JACQUES, S. M. Bioestatística: princípios e aplicações. Porto Alegre: Artmed, 2007, 255 p.

DROSTE, A.; SILVA, A. M.; MATOS, A. V.; ALMEIDA, J. W. In vitro propagation of Vriesea gigantea and Vriesea philippocoburgii: two vulnerable Bromeliads native to Southern Brazil. Arquivos de Biologia e Tecnologia, v. 48, n. 5, p. 717 - 722, 2005.

INSTITUTO BRASILEIRO DE GEOGRAFIA E ESTATÍSTICA (IBGE). Manual técnico da vegetação brasileira: sistema fitogeográfico, inventário das formações florestais e campestres, técnicas e manejo de coleções botânicas, procedimentos para mapeamentos. Rio de Janeiro: IBGE, 2 ed. 2012, 275 p.

INSTITUTO CHICO MENDES DE CONSERVAÇÃO DA BIODIVERSIDADE (ICMBIO). Floresta Nacional de São Francisco de Paula. Disponível em: <http://www.icmbio.gov.br/portal/biodiversidade/ unidades-de-conservacao/biomas-brasileiros/mata-atlantica/unidades-de-conservacao-mata-atlantica/2213 -flona-de-sao-francisco-de-paula.html> Acesso em: 10/05/2012.

JOHANSSON, D. Ecology of vascular epiphytes in west african rain forest. Acta Phytogeographica Suecica, v. 59, n. 5, p. 1 - 129, 1974.

KERSTEN, R. A.; KUNIYOSHI, Y. S. Conservação das florestas da bacia do Alto Iguaçu, Paraná Avaliação da comunidade de epífitas vasculares em diferentes estágios serais. Floresta, v. 39, n. 1, p. 51 66, 2009.

KERSTEN, R. A.; WAECHTER, J. L. Métodos quantitativos no estudo de comunidades epifíticas. In: Felfili, J. M.; Eisenlohr, P. V.; Melo, M. M. R. F.; Andrade, L. A.; Meira-Neto J. A. A. Fitossociologia no Brasil: métodos e estudos de caso. Viçosa: Editora UFV, v. 1, 2011. p. 156 - 176.

KRESS, W. J. The systematic distribution of vascular epiphytes: an update. Selbyana, v. 9, p. 2 - 22, 1986.

LORENZI, H. Árvores brasileiras: manual de identificação e cultivo de plantas arbóreas do Brasil., Nova Odessa: Instituto Plantarum, v. 1, 5 ed. 2008, 383 p.

MARTINELLI, G.; MORAES, M. A. Livro vermelho da flora do Brasil. 1 ed. Rio de Janeiro: Instituto de Pesquisas Jardim Botânico do Rio de Janeiro, 2013, 1102 p.

FLORESTA, Curitiba, PR, v. 45, n. 4, p. 781 - 790, out. / dez. 2015.

Becker, D. F. P. et al.

ISSN eletrônico 1982-4688 / ISSN impresso 0015-3826 
MEHLTRETER, K.; FLORES-PALACIOS, A.; GARCIA-FRANCO, J. Host preferences of low-trunk vascular epiphytes in a cloud Forest of Veracruz, Mexico. Journal of Tropical Ecology, v. 21, p. 651 660, 2005.

MISSOURI BOTANICAL GARDEN. TROPICOS.ORG. Disponível em: 〈http://www.tropicos.org> Acesso em: 10/03/2014.

MORAN, R. C.; KLIMAS, S.; CARLSEN, M. Low-trunk epiphytic ferns on tree ferns versus angiosperms in Costa Rica. Biotropica, v. 35, n. 1, p. 48 - 56, 2003.

PROCTOR, M. C. F. Light and desiccation responses of some Hymenophyllaceae (filmy ferns) from Trinidad, Venezuela and New Zealand: poikilohydry in a light-limited but low evaporation ecological niche. Annals of Botany, v. 109, p. 1019 - 1026, 2012.

REINERT, F.; FONTOURA T. Epiphytes. In: DEL CLARO, K.; OLIVEIRA, P. S.; RICO-GRAY, V. International Commission on Tropical Biology and Natural Resources. Paris: Encyclopedia of Life Support Systems (EOLSS), 2008. Disponível em: 〈http://www.eolss.net > Acesso em: 11/11/2012.

ROCHA, L. D.; DROSTE, A.; GEHLEN, G.; SCHMITT, J. L. Leaf dimorphism of Microgramma squamulosa (Polypodiaceae): a qualitative and quantitative analysis focusing on adaptations to epiphytism. Revista de Biologia Tropical, v. 61, p. 291 - 299, 2013.

SCHMITT, J. L.; WINDISCH, P. G. Aspectos florísticos e ecológicos de Alsophila setosa Kaulf. (Cyatheaceae, Pteridophita) no sul do Brasil. Acta Botanica Brasilica, v. 19, n. 4, p. 861 - 867, 2005.

SCHMITT, J. L.; WINDISCH, P. G.; BUDKE, J. C. Aspectos florísticos e ecológicos de pteridófitas epifíticas em cáudices de Dicksonia sellowiana Hook. (Pteridophyta, Dicksoniaceae), São Francisco de Paula, RS, Brasil. Pesquisas, Botânica, v. 56, p. 161 - 172, 2005.

SMYTH, N.; PHELAN, S.; DOUGLAS, G. Ex situ conservation of Trichomanes speciosum (Killarney fern) at the National Botanic Gardens and Kinsealy Teagasc Research Station. National Botanic Gardens, 2008. Disponível em: <http://www.botanicgardens.ie/conserve/trichomanes.htm> Acesso em: 25/09/2013.

FUNDAÇÃO SOS MATA ATLÂNTICA; INSTITUTO NACIONAL DE PESQUISAS ESPACIAIS (INPE). Atlas dos remanescentes florestais da Mata Atlântica e ecossistemas associados no período de 2000-2005. São Paulo, 2008, 156 p.

STRINGHETA, A. C. O.; SILVA, D. J. H.; CARDOSO, A. A.; FONTES, L. E. F.; BARBOSA, J. G. Germinação de sementes e sobrevivência das plântulas de Tilandsia geminiflora Brongn, em diferentes substratos. Acta Scientiarum, v. 27, p. 165 - 170, 2005.

TRYON, R. M.; TRYON, A .F. Ferns and allied plants with special reference to tropical america. New York: Springer-Verlag, 1982, 876 p.

WAGNER, K.; MENDIETA-LEIVA, G.; ZOTZ, G. Host specificity in vascular epiphytes: a review of methodology, empirical evidence and potentialmechanisms. AoB Plants, v. 7, p. 1 - 25, 2015. 